\title{
CONSIDERACIONES SOBRE LA FORMACIÓN EN LENGUA EXTRANJERA EN LA FUNDACIÓN UNIVERSITARIA DE CIENCIAS DE LA SALUD
}

Ricardo Augusto Gómez Becerra*

\section{Resumen}

El aprendizaje de una lengua extranjera pasa por procesos pedagógicos y evaluativos diferentes a los de cualquier otro saber académico o científico, ya que se trata de desarrollar competencias y no de adquirir un conocimiento específico. Este documento pretende mostrar a la comunidad académica de la Fundación los principios básicos del proceso educativo que el Centro de Idiomas ha implementado para todos los programas académicos, y al mismo tiempo intenta dar cuenta de la importancia del manejo de una segunda lengua en los procesos de formación de los futuros profesionales de las ciencias de la salud.

Palabras clave: marco común europeo, enfoque comunicativo, competencia lingüística.

\section{CONSIDERATIONS ABOUT LEARNING A FOREIGN LANGUAGE AT THE FUNDACIÓN UNIVERSITARIA DE CIENCIAS DE LA SALUD}

\section{Abstract}

Learning a foreign language involves particular pedagogic and evaluative processes that differ from those in learning any other academic or scientific information for it involves the development of competencies in contrast to acquiring a specific knowledge. This document pretends to show the FUCS academic community the basic principles of the educative process the Language Center has implemented for all academic programs, as well as, it intends to emphasize the importance the processes for learning a second language during training have for the future healthcare professionals.

Key words: Common European Framework [of Reference for Languages], communication-centered, linguistic competency.

\section{Antecedentes jurídicos y pedagógicos}

A comienzos de 2001, luego de años de análisis y debate sobre la problemática del bilingüismo por parte de docentes y representantes de diferentes sectores de la sociedad colombiana, el Ministerio de Educación Nacional formuló el Programa Nacional de Bilingüismo 2004 -
2019, en el que se incluyeron los estándares de competencia comunicativa en inglés con base en el marco común europeo. De esta manera, se establecieron referentes que posibilitaron, por primera vez, ver nuestros avances frente a otros países e introducir parámetros internacionales de medición al contexto local. Acorde con estas nuevas disposiciones, el objetivo del programa es lograr que los actores del sistema educativo desarrollen
Fecha recibido: agosto 24 de 2009 - Fecha aceptado: octubre 15 de 2009

* Docente Centro de Idiomas Fundación Universitaria de Ciencias de la Salud, Bogotá DC. Comunicador social y periodista, especialista en docencia universitaria. Certificado ICELT internacional de la Universidad de Cambridge, Reino Unido. 
competencias comunicativas en los niveles catalogados como intermedios, al tiempo que suficientes para su desempeño en el contexto internacional, como se observa en la Tabla 1.

\begin{tabular}{|l|c|}
\hline \multicolumn{2}{|c|}{ Tabla I. Niveles intermedios } \\
\hline Población & $\begin{array}{c}\text { Nivel de } \\
\text { lengua }\end{array}$ \\
\hline Docentes que enseñan inglés en la educación básica & $\mathrm{B} 2$ \\
\hline $\begin{array}{l}\text { Docentes de educación básica primaria y docentes de } \\
\text { otras áreas }\end{array}$ & $\mathrm{A} 2$ \\
\hline Estudiantes grado once & $\mathrm{B} 1$ \\
\hline Egresados de carreras en lenguas & $\mathrm{B} 2-\mathrm{Cl}$ \\
\hline Egresados de educación superior & $\mathrm{B} 2$ \\
\hline
\end{tabular}

A1 y A2 son niveles básicos en los que se busca comprender frases sencillas sobre tareas cotidianas. El nivel B1 busca que el educando entienda textos muy básicos sobre cuestiones que le sean conocidas, se desenvuelva en un viaje y sostenga conversaciones cortas, siempre y cuando el interlocutor hable despacio y muy bien pronunciado. El estudiante de nivel B2 puede entender textos no muy extensos que sean concretos o abstractos y posee un buen nivel conversacional. Puede argumentar para sentar posiciones o defender su punto de vista. El educando de nivel $\mathrm{C} 1$ puede comprender textos extensos y de mayor exigencia, así como producir escritos estructurados. Su nivel de comunicación es fluido y puede alternar sin ninguna dificultad con hablantes nativos. Ya en el nivel C2 se escribe y se habla con la fluidez del nativo. En concordancia con lo anterior, todas las instituciones académicas del país modificaron sus planes de estudio adoptando la enseñanza del inglés como lengua extranjera, dando así respuesta a la Ley 115 de 1994 que demanda "la adquisición de elementos de conversación, lectura, comprensión y capacidad de expresarse al menos en una lengua extranjera". En este proceso se han vinculado las secretarías de educación, universidades, centros de lenguas, organismos de cooperación internacional y proveedores de materiales educativos, entre otros.

La Fundación Universitaria de Ciencias de la Salud, por supuesto, no ha sido ajena a esta realidad que sirve de contexto a su actividad académica. Con la reciente creación del Centro de Idiomas, en junio de 2009, se unificaron en un solo organismo todas las actividades de formación en lenguas extranjeras que, hasta hace pocos años, venían desarrollando las facultades en forma aislada. Se constituyó un equipo docente de alta calidad y se reformaron los programas ajustándolos a los estándares planteados por el programa nacional de bilingüismo, de modo que sus estudiantes estuvieran en igualdad de condiciones con los egresados de todas las universidades del país. Sin embargo, la tarea es aún larga y difícil. Las condiciones socioculturales de nuestro país y las debilidades propias del sector educativo en sus modalidades básica y media, hacen que el conocimiento previo con que llegan los estudiantes a la universidad no sea óptimo para dar cumplimiento estricto a los estándares nacionales e internacionales de manejo de una segunda lengua. Nuestra condición de país hispanohablante impide que los estudiantes tengan exposición permanente a la lengua que están aprendiendo, lo cual es un factor clave para la práctica y apropiación de las habilidades lingüísticas.

El programa nacional de bilingüismo señala que en muchas instituciones del país, el aula y el entorno escolar son los únicos espacios disponibles para el uso del inglés y el tiempo de contacto de los estudiantes con el idioma es limitado. En la educación básica primaria algunas instituciones cuentan con una hora semanal para la enseñanza del inglés, aunque hay otras en las cuales todavía no se asigna tiempo. En la escuela secundaria y media la enseñanza del inglés cuenta con un promedio de dos a cuatro horas semanales.

Según el estudio Key Data on Teaching Languages at School in Europe, adelantado en 2005 por la Red de información de Educación Europea (Eurydice), en los 25 países de la Unión Europea, en el sistema educativo, la dedicación al estudio de una lengua extranjera es en promedio de ocho años, con una intensidad entre cinco y nueve horas semanales, desde los ocho años de edad del estudiante. En Colombia la dedicación promedio es de seis años, desde grado sexto a undécimo, a partir de los once años, con tres horas semanales. De acuerdo con el documento Bases para una Nación Bilingüe y Competitiva del Ministerio de Educación Nacional, un total de 720 horas para el estudio del inglés, durante la educa- 
ción básica y media, es suficiente para que los alumnos alcancen la competencia requerida en inglés.

A la luz de estas cifras, si bien el Ministerio de Educación es optimista, la disparidad es evidente. Mientras en los países europeos donde las posibilidades de interacción en inglés son más frecuentes, el estudiante cuenta con ocho años de formación en lengua extranjera y una intensidad horaria promedio de siete horas por semana, en nuestro país donde menos del $10 \%$ de la población es bilingüe los años de formación en inglés son dos menos y el tiempo semanal de formación en lengua extranjera se reduce casi a la mitad (cuatro horas como máximo). De este modo, aquí cualquier programa de formación en educación superior que contemple el desarrollo de la habilidad comunicativa en una lengua extranjera, debe contar con una intensidad horaria suficiente que de verdad permita a sus estudiantes un cierto dominio de la lengua.

La implementación de programas adecuados para formar profesionales con competencia comunicativa en lenguas extranjeras no se justifica, entonces, sólo desde el punto de vista legal ajustando los programas a las disposiciones del Ministerio de Educación Nacional, sino también desde una perspectiva holística que señala las condiciones mínimas para adquirir habilidades comunicativas suficientes. Ello, por supuesto, atañe a todos los programas académicos de la Fundación, pero en mayor medida a las facultades de medicina y enfermería cuyos egresados tienen altísima demanda en países angloparlantes.

Es un hecho que las políticas de bilingüismo se fundamentan en tres grandes pilares: lengua, comunicación y cultura, ya que el aprendizaje lingüístico es aprendizaje cultural. Si la Fundación quiere estar acorde con las exigencias de crecimiento del país es, entonces, necesario entender que la competencia comunicativa en lengua extranjera es mucho más que un curso libre o un requisito formal para la graduación de sus estudiantes. Es un compromiso fundamental con el desarrollo integral de los futuros profesionales de la salud, articulado con las demandas reales de formación que la vida nacional exige.

De acuerdo con el Ministerio de Educación Nacional ser bilingüe es esencial en un mundo globalizado. El manejo de una segunda lengua significa poderse comu- nicar mejor, abrir fronteras, comprender otros contextos, apropiar saberes y hacerlos circular, entender y hacernos entender, enriquecerse y jugar un papel decisivo en el desarrollo del país. Ser bilingüe es tener más conocimientos y oportunidades para ser más competentes y competitivos, y mejorar la calidad de vida de todos los ciudadanos.

\section{Importancia de la formación en lenguas extranjeras para los profesionales de las ciencias de la salud}

La educación bilingüe es una de las tendencias educativas más fuertes que en la actualidad se viven en el panorama educativo mundial y nacional. Cada vez hay más los programas de especialización y maestría que forman profesionales bilingües aptos para diseñar y desarrollar proyectos en lengua extranjera e, igualmente, es creciente el número de instituciones escolares que están ajustando sus programas a esta tendencia. En consecuencia, las oportunidades laborales de los egresados se multiplican cuando su perfil profesional se ajusta a la demanda de este campo de acción, aún naciente.

De manera similar, las posibilidades de hacer estudios de posgrado en el exterior aumentan de manera significativa cuando el egresado cuenta con un dominio adecuado de una lengua extranjera. Otra tendencia, aún más marcada en gran cantidad de universidades colombianas, es la exigencia de presentación de un examen internacional de competencia en lengua extranjera (TOEFL, IELTS, PET, FCE) como requisito para su graduación. En este sentido, la intensidad horaria y el grado de exigencia en el proceso formativo son altos.

Asimismo, otro campo que se abre frente las posibilidades específicas de los estudiantes y egresados de las carreras de la salud, es la realización de prácticas clínicas en convenios con universidades e instituciones médicas del extranjero. Es un hecho bien conocido por todos los profesionales del área que muchas de las instituciones médicas del mundo ofrecen, en forma permanente, excelentes oportunidades laborales para médicos, enfermeros e instrumentadores, pero la oferta de profesionales de la salud bilingües - y al mismo tiempo for- 
mados en un campo de especialización concreto-es limitada o no está bien calificada. Por otra parte, el convulsionado panorama mundial, la crudeza de los conflictos contemporáneos y los grandes avances en materia científica demandan cada vez más profesionales e investigadores bilingües en todas las áreas del conocimiento, aptos para pensar el problema de la salud y de las ciencias en el marco de la multiculturalidad y la coexistencia de las razas.

Sólo optimizando los programas de lengua inglesa de la Fundación en términos de tiempos de formación y exposición a la lengua extranjera, grado de exigencia en el aula de clases y competencias mínimas requeridas para la graduación acorde con los estándares internacionales, los egresados de los programas de ciencias de la salud estarán en capacidad de enfrentar de manera competitiva estas demandas, ofreciendo soluciones adecuadas a las problemáticas científicas del mundo contemporáneo.

En la Fundación, desde hace dos años la Oficina de Aseguramiento de la Calidad, después la Oficina de Orientación Universitaria y ahora bajo la dirección de la Vicerrectoría de Planeación y Proyectos Especiales, han venido trabajando conjuntamente con el fin de consolidar los recursos humanos y tecnológicos necesarios para garantizar una formación de calidad en lenguas extranjeras para los estudiantes y demás miembros de la comunidad académica. Como resultado surgió el Centro de Idiomas, que poco a poco empieza a posicionarse en el panorama educativo de la Fundación junto a las facultades y demás unidades académicas de la universidad.

Del mismo modo, con el ánimo de aprovechar las ventajas de contar con un centro especializado en lenguas para reforzar la formación de los estudiantes de los programas en ciencias de la salud, a partir del segundo semestre de 2009 se implementó un programa específico en inglés técnico para enfermería y pronto habrá uno de inglés técnico para medicina, basados en el enfoque pedagógico English for Specific Purposes, de modo que la formación ofrecida sea más ajustada a la demanda de un mercado aún inexplorado en nuestro país y que pondrá a la Fundación a la vanguardia de las instituciones formadoras de profesionales de la salud, con un Centro de Idiomas especializado en Ciencias de la Salud.
Los resultados obtenidos hasta el momento son halagüeños no sólo por el desempeño lingüístico de los estudiantes en lengua extranjera, que ha aumentado en forma significativa en relación con el de promociones anteriores a 2009, sino por el fortalecimiento de la malla curricular de los diferentes programas formativos de la Fundación.

A partir de la consolidación de un equipo docente encargado de la formación en lengua extranjera para la universidad, se ha logrado una mayor coherencia de los programas académicos en concordancia con los lineamientos del Programa Nacional de Bilingüismo, se espera ampliar sustancialmente las oportunidades de nuestros estudiantes para ser aceptados en prácticas profesionales en instituciones médicas y hospitalarias angloparlantes, ha mejorado la perspectiva de acción profesional de nuestros egresados dada la importancia que la Fundación le ha dado a la formación en lengua extranjera y, en consecuencia, se ha elevado su perfil profesional lo que constituye una evidente ventaja competitiva frente a egresados de otras universidades.

Es mucho, sin embargo, lo que queda por hacer en materia de educación en lenguas extranjeras para los trabajadores del área de la salud. Pese a su evidente importancia con miras a la optimización del perfil de los profesionales, en nuestro país sigue habiendo una evidente separación entre los procesos formativos que desarrollan las facultades de medicina o enfermería, para citar sólo algunas, y la demanda efectiva de profesionales bilingües en estas áreas. El manejo de una segunda lengua sigue siendo entera responsabilidad del individuo y es hora de que los centros de formación en ciencias de salud asuman esta tarea. En este sentido, la Fundación ya ha empezado a dar sus primeros pasos en firme, de modo que podamos garantizar que nuestros egresados tengan la competencia lingüística en lengua extranjera, necesaria para aportar en forma más efectiva al progreso y la cambiante realidad de nuestro país.

\section{Lecturas recomendadas}

Bases para una Nación Bilingüe y Competitiva. (s.f.). Artículo en línea, en: http:/ /www.mineducacion.gov.co/1621/article-97498.html. Consultado el 20 de julio de 2009.

Ministerio de Educación Nacional - República de Colombia (2006). Formar en Lenguas Extranjeras: Inglés ¡El Reto! Bogotá: Imprenta Nacional. 